\title{
Immunohistochemical detection of the lymphatic marker podoplanin in diverse types of human cancer cells using a novel antibody
}

\author{
TOMOYA KONO ${ }^{1,2^{*}}$, MASAYUKI SHIMODA ${ }^{1,3^{*}}$, MEIKO TAKAHASHI $^{1}$, KYOICHI MATSUMOTO $^{4}$, \\ TAKANOBU YOSHIMOTO ${ }^{1}$, MIKA MIZUTANI ${ }^{1}$, CHIHARU TABATA $^{1}$, \\ $\mathrm{KAE} \mathrm{OKOSHI}^{1,3}$, HIROMI WADA ${ }^{2}$ and HAJIME KUBO ${ }^{1}$ \\ ${ }^{1}$ Molecular and Cancer Research Unit, HMRO, Departments of ${ }^{2}$ Thoracic Surgery, \\ ${ }^{3}$ Surgery, Graduate School of Medicine, Kyoto University, Kyoto; \\ ${ }^{4}$ Japan Clinical Laboratories, Inc., Kyoto, Japan
}

Received March 26, 2007; Accepted May 9, 2007

\begin{abstract}
The mucin-type transmembrane glycoprotein podoplanin (also known as T1 $\alpha$, gp38 or Aggrus) is well established as one of the lymphatic-specific markers. Podoplanin was also reported to be associated with tumorinduced platelet aggregation and tumor metastasis. Here, we generated a novel monoclonal antibody (clone; 7B10) that specifically recognized human podoplanin, as assessed by enzyme-linked immunosorbent assay, Western blot analyses, immunohistochemistry and flow cytometry. 7B10 stained not only lymphatic vessels but also type I alveolar cells in the lung, podocytes in the kidney and myoepithelial cells in the breast. In addition, podoplanin expression was analyzed by immunostaining on tissue microarrays that included 12 different cancer types using 7B10. Consequently, we found that podoplanin was expressed by cancer cells derived from esophagus, lung, liver, colon and breast, as well as lymphatic endothelial cells. These findings suggest a potential role of podoplanin in tumor progression in diverse types of human cancers.
\end{abstract}

Correspondence to: Dr Hajime Kubo, Molecular and Cancer Research Unit, HMRO, Graduate School of Medicine, Kyoto University, Yoshida Konoe-cho, Sakyo-ku, Kyoto 606-8501, Japan E-mail: kuboflt@kuhp.kyoto-u.ac.jp

Present address: Department of Surgery, Graduate School of Medicine, Kyoto University, Shogoin-Kawahana-cho, Sakyo-ku, Kyoto 606-8507, Japan

${ }^{*}$ Contributed equally

Abbreviations: SCC, squamous cell carcinoma; ELISA, enzymelinked immunosorbent assay; mAb, monoclonal antibody; LECs, lymphatic endothelial cells; HRP, horseradish peroxidase; OPD, o-phenylendiamine

Key words: podoplanin, lymphatic marker, antibody, immunohistochemistry, tissue microarray

\section{Introduction}

The mucin-type transmembrane glycoprotein podoplanin (also known as T1 $\alpha$, gp38 or Aggrus) is specifically expressed by lymphatic but not blood vascular endothelial cells, and thus is well established as one of the lymphatic-specific markers $(1,2)$. During embryogenesis, $\mathrm{T} 1 \alpha$ /podoplanin is expressed by the mouse foregut endoderm before the lung buds at embryonic day E8.5-9.0 $(3,4)$. Homozygous null mutant mice die at birth due to respiratory failure. $\mathrm{T} 1 \alpha /$ podoplanin $^{-/-}$mice also have defects in the development of lymphatic vasculature, leading to dilation of cutaneous and intestinal lymphatic vessels, and congenital lymphedema. However, little is understood about the biological function of podoplanin in the formation of lymphatic vasculature, i.e. lymphangiogenesis. In pathological conditions, several lines of evidence, obtained from mouse tumor models and analyses of human cancer samples, have suggested that tumor lymphangiogenesis promotes lymphatic tumor spread (5-7).

Aggrus/podoplanin was reported to possess the ability of inducing platelet aggregation. Interaction between tumor cells and platelets have been considered to facilitate the arrest of tumor cell cluster in the microcirculation and the subsequent development of experimental metastasis $(8,9)$. The 8F11 monoclonal antibody was originally established to inhibit platelet aggregation in vitro and the pulmonary metastasis of highly metastatic clones of mouse colon adenocarcinoma cell line (Colon-26) in vivo (10-13). Recent reports have demonstrated that $8 \mathrm{~F} 11$ specifically recognize mouse Aggrus/podoplanin and that purified Aggrus/ podoplanin protein induced platelet aggregation without plasma components $(14,15)$. Aggrus/podoplanin expression might be associated with tumor-induced platelet aggregation and tumor metastasis. Previously, podoplanin expression was largely restricted to specific types of malignancies, such as testicular germ cell tumors and peritoneal mesotheliomas $(16,17)$. However, podoplanin was subsequently found in colorectal tumors, skin squamous cell carcinoma (SCC) and lung SCC $(15,18,19)$. 
In this study, we generated a novel monoclonal antibody (mAb) (clone; 7B10) against human podoplanin whose specificity was assessed by enzyme-linked immunosorbent assay (ELISA), Western blotting, immunohistochemistry and flow cytometry, and screened the expression of podoplanin both in normal tissues and in different types of cancers by immunostaining using 7B10. For this purpose, we utilized tissue microarrays, including 120 different normal tissues and several types of cancer samples from 12 different tumor categories. Our results suggest that podoplanin expression is found in diverse types of human cancer cells, and that podoplanin may not only function as a specific diagnostic marker for a subset of malignancies, but may also be associated with tumor progression in diverse types of human cancers.

\section{Materials and methods}

Human samples. Paraffin-embedded formalin fixed tissues were retrieved from the 2000-2004 surgical pathology files of Kyoto University Hospital. All samples were obtained with informed consent and their use was approved by the ethics committee of the hospital. Tissue arrays of human normal tissues (human tissue array MAN2, MBN2) and tumors (human tissue array MA2, MB2) were purchased from SuperBioChips (Korea).

Cell culture. 293T cells and NIH3T3 cells were cultured in DMEM supplemented with $10 \%$ FCS (JRH). Human lymphatic endothelial cells (LECs) were purchased from AngioBio (Del Mar, CA), and maintained in EGM-2 MV (Cambrex, Walkersville, MD) supplemented with $20 \%$ off-clot human serum (OYC, Tokyo, Japan).

Plasmids. Total RNA was isolated from cultured human LECs using TRIzol Reagent (Gibco), and subsequently digested with RNase-free DNase I (Roche, Mannheim, Germany) and tested for integrity. Total RNA (10 $\mu \mathrm{g})$ was used to synthesize cDNA by using SuperScript II reverse transcriptase (Invitrogen) and oligo-dT primers. Full length human podoplanin (GenBank accession AF390106) was amplified from $1 \mu \mathrm{l}$ of the cDNA by polymerase chain reaction with the primers; 5'-TAATAAGCTTATGTGGAAGGTGT CAGCTCT-3' and 5'-TATATCTAGAGGGCGAGTAC CTTCCCGACATTTTT-3' using ExTaq DNA polymerase (Takara). The HindIII-XbaI fragment was subcloned into pcDNA/V5-His-A (pcDNA-podoplanin-V5). Next, a 489-bp fragment of human podoplanin cDNA (positions 1-489 in GenBank AB127958), encoding the extracellular domain of podoplanin, was subcloned into the expression plasmid pIgplus, to yield a construct encoding podoplanin fused at the C-terminus to the Fc region of human IgG (pIgpluspodoplanin)

Generation of anti-human podoplanin antibodies. 293T cells were transfected with pIgplus-podoplanin using FuGENE6 (Roche), and cells were cultured in DMEM without FCS for 5 days before harvesting culture supernatants. Podoplanin-Fc fusion proteins were purified by affinity chromatography on a column of $1 \mathrm{ml}$ of protein A Sepharose (Amersham Pharmacia Biotech AB, Uppsala, Sweden).
Mouse monoclonal antibodies against the human podoplaninFc fusion protein were generated as described previously with some modification (20). In brief, 8-week-old mice were first immunized subcutaneously with $20 \mu \mathrm{g}$ podoplanin-Fc protein in complete Freund's adjuvant (Difco, Detroit, MI), followed by three times intraperitoneal injections of $20 \mu \mathrm{g}$ podoplanin-Fc protein in Freund's incomplete adjuvant in alternating weeks and a final intravenous boost of $5 \mu \mathrm{g}$ podoplanin-Fc protein. Three days after the boost, spleen cells were harvested and fused with mouse myeloma P3U1 cells. Undiluted supernatants from hybridomas were screened by ELISA plates coated with $50 \mathrm{ng} / \mathrm{ml}$ podoplanin-Fc protein. Positive hybridomas were cloned using the limiting dilution technique. To select monoclonal hybridomas reacting positively to podoplanin-Fc protein, binding was detected by horseradish peroxidase (HRP) conjugated goat anti-mouse IgG $(\mathrm{H}+\mathrm{L}) \mathrm{Ab}$. Plate-bound enzymic activity was detected by OPD (o-phenylendiamine) substrate-chromogen and absorbance of each well was measured using a microplate reader.

Immunofluorescence staining. Immunostaining of LECs on dishes was performed as follows. Cells were fixed by $4 \%$ PFA/PBS for 10 min on ice. After washing with PBS, cells were blocked with $1 \% \mathrm{BSA} / \mathrm{PBS}$ for $30 \mathrm{~min}$ at room temperature. The fixed cells were incubated with culture supernatants obtained from selected hybridomas or purified 7B10 mAb, followed by donkey anti-mouse Alexa Fluor 488 Ab (1:1000, Molecular Probes, OR).

Transfection and Western blot analysis. Subconfluent NIH3T3 cells were transfected with pcDNA3-podoplanin-V5 ( $1 \mu \mathrm{g}$ DNA per $10-\mathrm{cm}$ cell culture plate) or pcDNA3-V5 as a control using FuGENE 6 according to manufacturer's instructions, and were selected in $800 \mu \mathrm{g} / \mathrm{ml} \mathrm{G} 418$ (Sigma) for 10 days. For Western blotting, cells were lysed in DOCRIPA lysis buffer (50 mM Tris- $\mathrm{HCl} \mathrm{pH} 8.0,150 \mathrm{mM} \mathrm{NaCl}$, $1 \%$ Triton $\mathrm{X}-100,0.1 \%$ SDS, $1 \%$ DOC, 10 mM EDTA) supplemented with aprotinin, leupeptin, PMSF and sodium vanadate. The total cell lysates were separated in $12.5 \%$ SDS-PAGE. The blots were probed with mouse anti-podoplanin $\mathrm{mAb}$ (clone; 7B10, $5 \mu \mathrm{g} / \mathrm{ml}$ ) and HRP-conjugated anti-mouse secondary antibodies (1:5000, Dako, Glostrup, Denmark), followed by detection by the ECL method (Western Lightning Chemiluminescence Reagent Plus, Perkin-Elmer Life Sciences, Boston, MA). Filters were reprobed with antiV5 mAb (1:1000, Invitrogen). The total cell lysates from LECs were loaded as a control.

Flow cytometry. Flow cytometric analysis was performed by FACS Calibur (Becton-Dickinson). Cultured cells were harvested and dissociated with $0.25 \%$ trypsin-EDTA (Gibco) treatment. The cells were stained with a combination of mAbs of 7B10 (5 $\mu \mathrm{g} / 10^{6}$ cells $\left./ \mathrm{ml}\right)$ and goat anti-human VEcadherin $\left(0.1 \mu \mathrm{g} / 10^{6}\right.$ cells $/ \mathrm{ml}, \mathrm{R} \& \mathrm{D}$ Systems, MN) followed by incubation with donkey anti-mouse Alexa Fluor 488 (1:1000, Invitrogen) and rabbit anti-goat Alexa Fluor 633 (1:1000, Invitrogen).

Immunohistochemistry. The paraffin-embedded sections were deparaffinized and hydrated by standard methods (21). 
After antigen retrieval with microwave (600 W 5 min and $200 \mathrm{~W} 10 \mathrm{~min}$ ) in sodium citrate, endogenous peroxidase was quenched with $3 \% \mathrm{H}_{2} \mathrm{O}_{2}$ in methanol for $10 \mathrm{~min}$. After blocking with TNB buffer (TSA Biotin system kit/NEL700, Perkin-Elmer Life Science) for $30 \mathrm{~min}$, sections were exposed to primary antibodies [7B10,1 $\mu \mathrm{g} / \mathrm{ml}$; rat anti-CD144 $\mathrm{Ab}$ (VE-cadherin), 1:100, PharMingen; rabbit anti-LYVE-1 Ab, 1:100] in TNB for $16 \mathrm{~h}$ at $4^{\circ} \mathrm{C}$, washed with TNT buffer and incubated $30 \mathrm{~min}$ at room temperture with biotinylated secondary antibodies (Dako) diluted in TNB. The tyramide signal amplification was used to enhance staining. Peroxidase activity was developed with EnVision kit/HRP (DAB) (Dako) and the sections were counterstained with hematoxylin. In negative control stains, the primary antibodies were omitted. The staining intensity was estimated for each cell type on a three-step scale $(-,+,++)$ by two investigators.

\section{Results}

Production and characterization of anti-human podoplanin $m A b s$. After screening more than 1000 clones by ELISA for the immunoreaction to podoplanin-Fc protein, 25 monoclonal clones were isolated. To select clones available for immunohistochemistry, we performed immunofluorescence staining of LECs by the undiluted conditioned medium from 25 hybridomas and found that 7B10 intensely stained LECs (Fig. 1a). To evaluate the specificity of 7B10, we transfected NIH3T3 cells, which express no endogenous podoplanin, with human full-length podoplanin or empty vector, and established the NIH3T3/podoplanin cells and NIH3T3/mock cells respectively. The band consistent with the size of human podoplanin protein $(43 \mathrm{kDa})$ was immunodetected with $7 \mathrm{~B} 10$ in the total cell lysates from NIH3T3/podoplanin and LECs, but not from NIH3T3/mock, as assessed by Western blotting (Fig. 1b). Flow cytometric analysis also demonstrated that $7 \mathrm{~B} 10$ reacted with NIH3T3/podoplanin but not with NIH3T3/mock (Fig. 1c) and that LECs were stained by both 7B10 and by an endothelial marker, VE-cadherin (Fig. 1d).

Podoplanin expression in lymphatics. We investigated by immunostaining whether 7B10 could detect lymphatic vessels in comparison with the expressions of another specific lymphatic marker, LYVE-1 and panendothelial marker, VE-cadherin. In serial sections of normal stomach tissues, 7B10 stained endothelial cells of the vessels devoid of red blood cells that were also positive for LYVE-1 and VE-cadherin, suggesting 7B10 specifically detected lymphatics (Fig. 2a-c). Numerous VE-cadherin ${ }^{+}$LYVE-1vessels, characteristic of blood vessels, were negative for podoplanin, confirming specific podoplanin expression by lymphatics, but not by blood vessels. We next studied whether 7B10 might also stain lymphatics in other human organs and in pathological conditions. In all human organs examined using human tissue microarrays including 120 different normal tissues, we found positive staining on lymphatics by 7B10. Several lymphatic vessels stained by $7 \mathrm{~B} 10$ were observed in the mucosa and the submucosa of intestine (Fig. 2d). According to previous reports $(22,23)$, sinusoidal blood vessels in the liver expressed lymphatic markers VEGFR3 and LYVE-1, but they were not positive for podoplanin, as assessed by immunostaining using 7B10 (data not shown). Thus, podoplanin staining is a good means to identify lymphatic vessels in the liver. Interestingly, in the sections of cirrhotic liver obtained from Kyoto University Hospital, increased lymphatic vessels were found within the fibrotic tissues (Fig. 2e). In addition, in all types of malignancies examined including lung cancers, lymphatics were detected by 7B10 (Fig. 2f). Taken together, we concluded that 7B10 was specific for podoplanin and can be used for immunohistochemistry, and used 7B10 as antihuman podoplanin $\mathrm{mAb}$ in the following studies.

Podoplanin expression in normal tissues. We next asked whether other cell types would express podoplanin using human multiple tissue arrays. As previously reported $(3,4,19,24)$, podoplanin was expressed by alveolar type I cells in the lung and by podocytes and glomerular capsule epithelium in the kidney (Fig. 3a-d). Moreover, podoplanin expression was observed on myoepithelial cells of the mammary glands (Fig. 3e and f).

Podoplanin expression in diverse types of cancer cells. 7B10 immunostained podoplanin was expressed in the membrane of both epithelial and sarcomatous mesothelioma (Fig. 4a and b). 7B10 also demonstrated the expression of podoplanin in the lung cancers (Fig. 4c and d). In comparison with mesotheliomas, the localization of podoplanin in lung cancers was not restricted to the membrane. The finding was compatible with a previous report (19). We then investigated whether tumor cells themselves might express podoplanin using tissue microarrays, including cancer samples from 12 different tumor categories. The results of our comparable tumor analyses are shown in Table I. As previous reports suggested that podoplanin expression was restricted to specific types of malignancies, such as testicular germ cell tumors and peritoneal mesotheliomas, or SCCs in the skin and lung (16-19), podoplanin was expressed by the majority of SCCs of the esophagus (7 of 10 tumors, Fig. 4e) and those of the lung studied (11 of 13 tumors, Fig. 4g). In contrast, podoplanin was absent from adenocarcinomas of esophagus $(n=1)$, stomach $(n=10)$, ovary $(n=10)$, pancreas $(n=10)$ and prostate $(n=8)$ (Table I; Fig. 4f, o and p). However, various levels of podoplanin expression were also seen in some adenocarcinomas of lung, colon, thyroid, breast, liver and kidney (Fig. 4). Of 8 lung carcinomas, 1 tumor was strongly positive, 3 were moderately positive and 4 were negative (Fig. $4 \mathrm{~g}$ and h). Importantly, the cancerous tissues contained normal lung tissues, and only these normal tissues comprising of alveolar type I cells were strongly stained (Fig. 4h). Of 10 colon adenomas, 4 tumors were strongly positive, 4 were moderately positive and 1 was negative, consistent with previous reports (Fig. 4i and j) (15). Of 10 breast carcinomas, 1 tumor was strongly positive, 3 were moderately positive and 6 were negative (Fig. $4 \mathrm{~m}$ and $\mathrm{n}$ ). Moreover, all thyroid carcinomas (9 of 9 tumors) studied were moderately stained for podoplanin, whereas the normal follicle epithelium was not stained (Table I and data not shown). Interestingly, a liver tumor with a combined hepatocellular and cholangiocarcinoma showed hetero- 
a

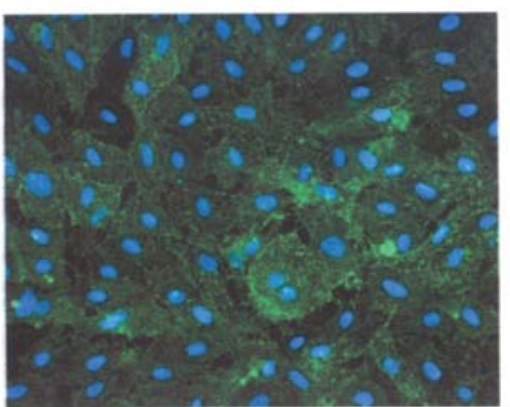

c

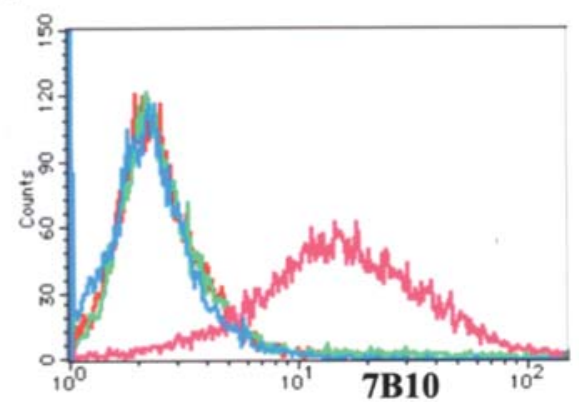

b

kDa

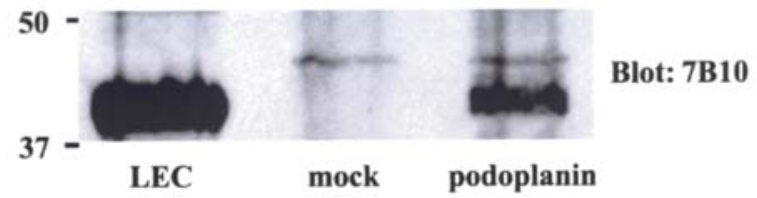

d

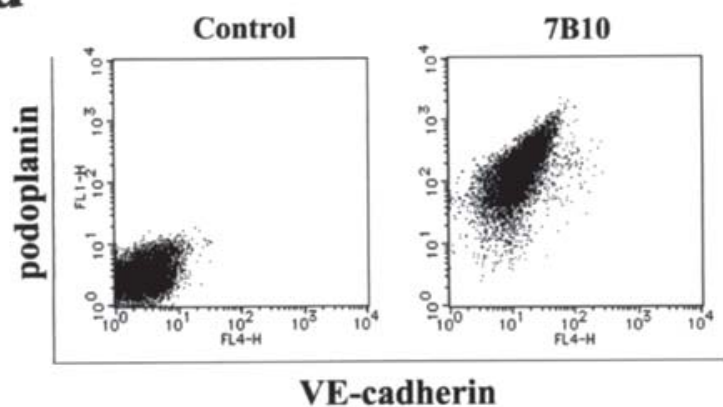

Figure 1. Production and characterization of anti-human podoplanin mAbs. (a) Immunostaining of the cultured lymphatic endothelial cells (LECs) with antihuman podoplanin mAb (clone; 7B10). Green, 7B10; Blue, Hoechst (b) Western blotting of LECs with 7B10 mAb, NIH3T3 mock transfectant; NIH3T3/mock (mock), and NIH3T3 transfected with the full-length human podoplanin; NIH3T3/podoplanin (podoplanin). (c) Flow cytometric analysis using 7B10 mAb of NIH3T3/podoplanin in comparison with NIH3T3/mock. Green, control; Purple, NIH3T3/mock; Red, NIH3T3/podoplanin. (d) Flow cytometric analysis of LECs with 7B10 in combination of anti-VE-cadherin antibody.

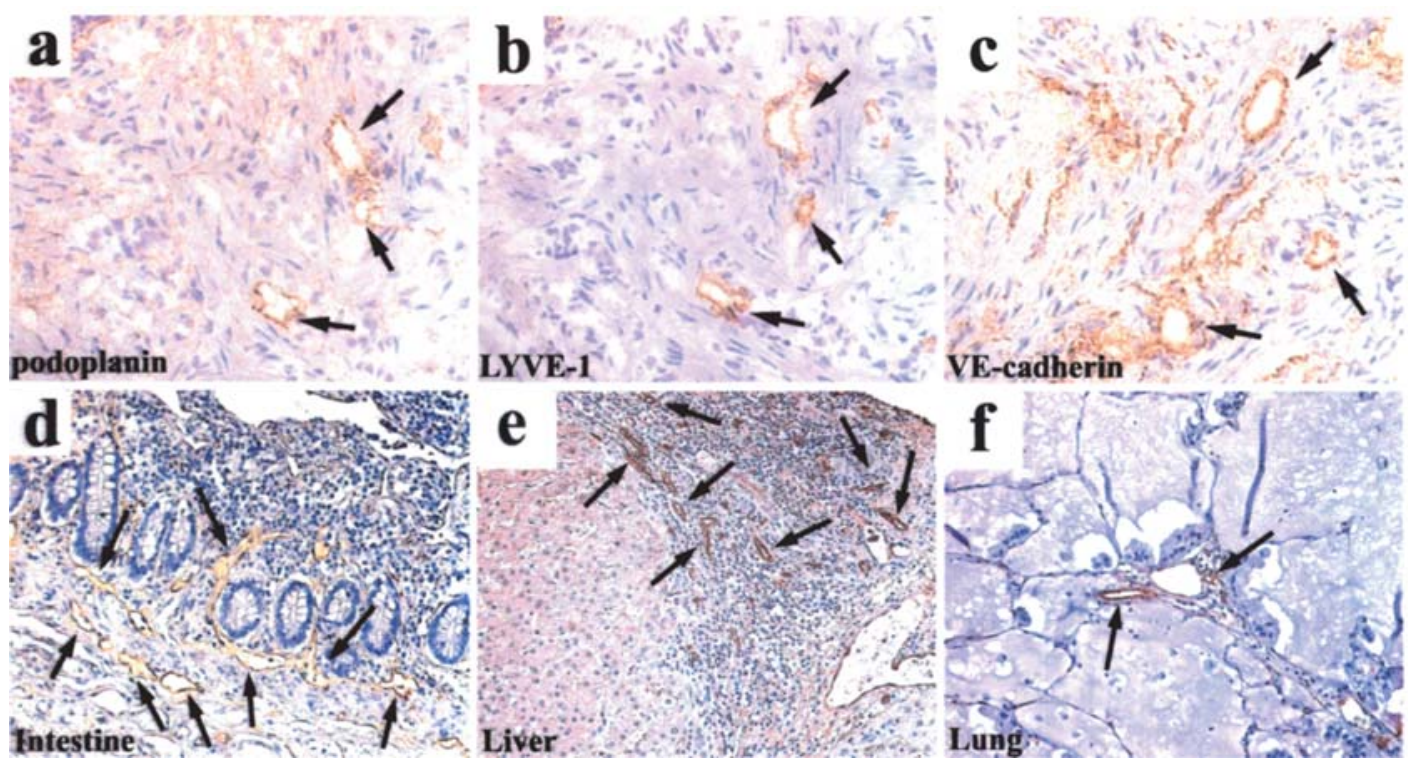

Figure 2. Podoplanin expression in lymphatics. Immunostaining of serial sections obtained from the normal stomach with 7B10 (a), anti-LYVE-1 Ab (b) and anti-VE-cadherin mAb (c). The lymphatics stained by 7B10 in the normal intestine (d), the cirrhotic liver (e) and the lung cancer (f). Arrows indicate lymphatics. Original magnification: x400 (a-c), x200 (d-f).

geneous pattern of staining (Fig. 4k), while 7 of 8 hepatocellular carcinomas were negative. In addition, we found podoplanin expression in kidney cancers (4 of 8 tumors) and urine cancers ( 1 of 10 tumors). These results suggested that podoplanin expression can be found in diverse types of human cancer cells.

\section{Discussion}

The findings we present herein challenge the prevailing view that the detection of podoplanin expression is a good means of identifying lymphatic vessels. We established a novel mouse anti-human podoplanin mAb (7B10) available for immunohistochemistry and found that podoplanin was also 


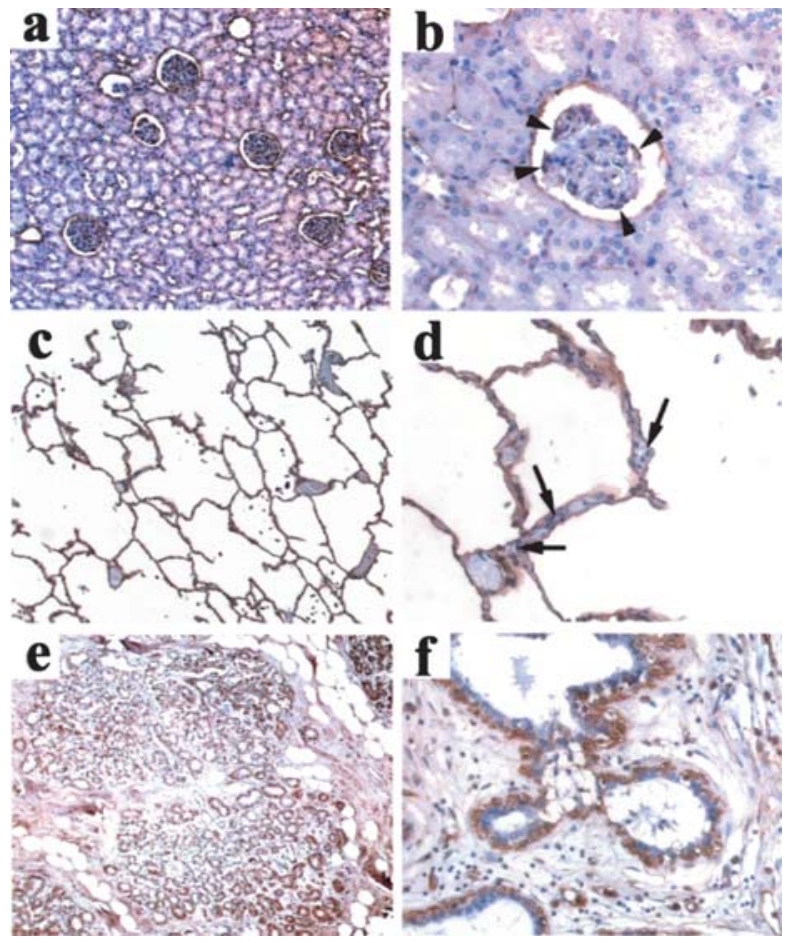

Figure 3. Podoplanin expression in normal tissues. Podoplanin expression, as assessed by 7B10 immunostaining, was observed in the normal tissues besides lymphatics. Type I alveolar cells were stained in the normal lung ( $\mathrm{a}$ and $\mathrm{b}$ ). Bowman capsule epithelium and podocytes were stained in the kidney (c and d). In the breast, myoepithelial cell lining outside the mammary epithelial cells were strongly stained (e and f). Original magnification: x100 (a, c and e), x200 (b, d and f). expressed by diverse types of cancer cells. Contrary to the published reports (16-19), podoplanin expression was not restricted to specific types of malignancies, such as testicular germ cell tumors and peritoneal mesotheliomas, nor to specific histological types of tumors, SCCs. The finding that colon carcinomas largely expressed podoplanin was consistent with a previous report (15). Indeed, according to this study demonstrating that podoplanin was also detected in several types of carcinomas as well as colon carcinomas, we may have to evaluate the expression levels of podolanin in association with large clinical follow-up data. In the analysis of the limited clinical follow-up data studied here, podoplanin expression may be associated with nodal positivity (data not shown).

Podoplanin has putative extracellular and transmembrane domains and a short cytoplasmic tail with putative protein kinase $\mathrm{C}$ and cAMP phosphorylation sites. Sequence analysis indicates that podoplanin does not share common domains with other protein families of known function that predict its function (15). Although several functions were suggested based on the expression patterns, the physiological role of podoplanin has not yet been determined. Podoplanin was first identified as an early response protein (OTS-8) that was induced by phorbol ester in osteoblastic cells (25). Mouse podoplanin has also been reported in thymus epithelial cells and in peripheral lymphoid tissues $(26,27)$. Developmental expression patterns and the analyses of podoplanin -/- mice indicate that podoplanin might play a role in the formation of

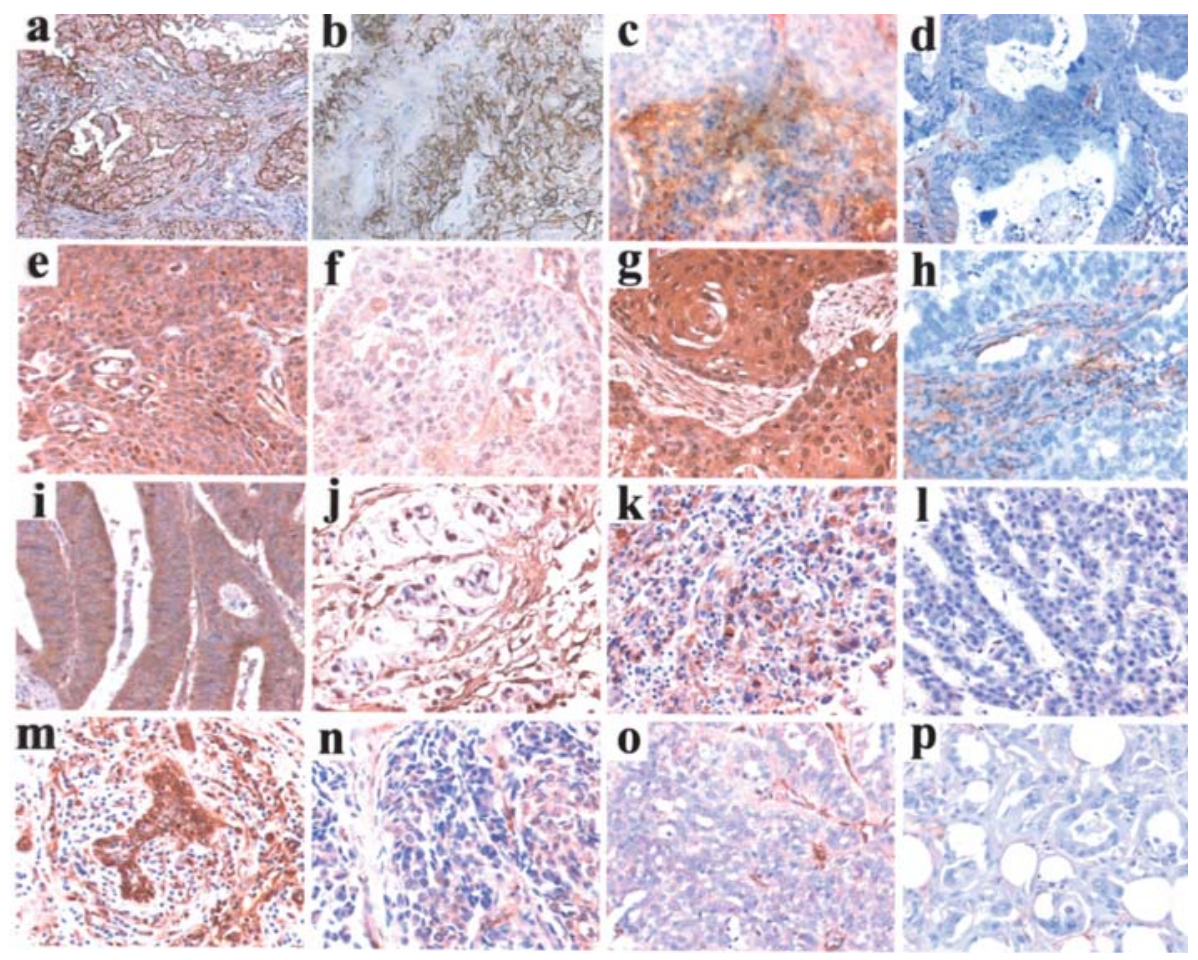

Figure 4. Podoplanin expression in diverse types of cancer cells. (a-d) Immunostaining for podoplanin in epithelial (a) or sarcomatous (b) mesothelioma and lung cancers (c, squamous cell carcinoma; d, adenocarcinoma). (e-p) The analysis of the podoplanin expression in cancer tissues by $7 \mathrm{~B} 10$ immunostaining using tissue microarrays, including cancer samples from 12 different organs. Note the podoplanin expression by cancer cells. Representative stains, including negative stains, are shown in cancers from esophagus (e); squamous cell carcinoma, (f); infiltrating squamous cell carcinoma, lung (g); squamous cell carcinoma, (h), adenocarcinoma, colon (i); adenocarcinoma, (j); mucinous adenocarcinoma, liver (k); combined hepatocellular and cholangiocarcinoma, (l); hepatocellular carcinoma, breast (m); infiltrating duct carcinoma, (n); infiltrating papillary carcinoma with signet ring cell carcinoma, ovary (o); serous cystoadenocarcinoma and prostate (p); adenocarcinoma). Original magnification: $\mathrm{x} 400$. 
Table I. Podoplanin expression in human tumors.

\begin{tabular}{|c|c|c|c|c|}
\hline & $\begin{array}{c}- \\
\text { Cases }(\%)\end{array}$ & $\begin{array}{c}+ \\
\text { Cases }(\%)\end{array}$ & $\begin{array}{c}++ \\
\text { Cases }(\%)\end{array}$ & $\begin{array}{c}\text { Total } \\
\text { no. }\end{array}$ \\
\hline Stomach & $10(100)$ & $0(0)$ & $0(0)$ & 10 \\
\hline Adenocarcinoma & 9 & & & 9 \\
\hline Mucinous adenocarcinoma & 1 & & & 1 \\
\hline Esophagus & $3(30)$ & $2(20)$ & $5(50)$ & 10 \\
\hline Adenocarcinoma & 1 & & & 1 \\
\hline Squamous cell carcinoma & 1 & 2 & 4 & 7 \\
\hline Infiltrating squamous cell carcinoma & 1 & & & 1 \\
\hline Basaloid squamous cell carcinoma & & & 1 & 1 \\
\hline Lung & $6(27)$ & $10(45)$ & $6(27)$ & 22 \\
\hline Large cell carcinoma & & 1 & & 1 \\
\hline Squamous cell carcinoma & 2 & 6 & 5 & 13 \\
\hline Adenocarcinoma & 4 & 3 & 1 & 8 \\
\hline Colon & $2(20)$ & $4(40)$ & $4(40)$ & 10 \\
\hline Adenocarcinoma & 1 & 4 & 4 & 9 \\
\hline Mucinous adenocarcinoma & 1 & & & 1 \\
\hline Thyroid & $0(0)$ & $9(100)$ & $0(0)$ & 9 \\
\hline Papillary carcinoma & & 9 & & 9 \\
\hline Kidney & $4(50)$ & $3(38)$ & $1(13)$ & 8 \\
\hline Renal cell carcinoma & 4 & 2 & 1 & 7 \\
\hline Nephrogenic carcinoma & & 1 & & 1 \\
\hline Breast & $6(60)$ & $3(30)$ & $1(10)$ & 10 \\
\hline Infiltrating duct carcinoma & 3 & 2 & 1 & 6 \\
\hline Infiltrating papillary carcinoma & 2 & & & 2 \\
\hline Medullary carcinoma & 1 & 1 & & 2 \\
\hline Liver & $8(80)$ & $2(20)$ & $0(0)$ & 10 \\
\hline Hepatocellular carcinoma & 7 & 1 & & 8 \\
\hline Combined & 1 & 1 & & 2 \\
\hline Urinary bladder & $9(90)$ & $1(10)$ & $0(0)$ & 10 \\
\hline Transitional cell carcinoma & 8 & 1 & & 9 \\
\hline Mucinous adenocarcinoma & 1 & & & 1 \\
\hline Ovary & $10(100)$ & $0(0)$ & $0(0)$ & 10 \\
\hline Serous cystoadenocarcinoma & 9 & & & 9 \\
\hline Mucinous cystoadenocarcinoma & 1 & & & 1 \\
\hline Pancreas & $10(100)$ & $0(0)$ & $0(0)$ & 10 \\
\hline Ductal adenocarcinoma & 10 & & & 10 \\
\hline Prostate & $7(88)$ & $1(13)$ & $0(0)$ & 8 \\
\hline Adenocarcinoma & 7 & 1 & & 8 \\
\hline
\end{tabular}

bronchial tree or lymphatic system. In the adult, podoplanin could be a regulator of fluid transport, since it is expressed by a number of tissues involved in fluid fluxes and is coexpressed with some aquaporins (19). Importantly, it has recently been reported that podoplanin is identical to Aggrus, which is already known to function as an adhesion molecule that promotes platelet aggregation in pathological conditions and consequently metastatic spread in colorectal tumors (15). In agreement with the report, our findings indicated that podoplanin may not only function as a specific diagnostic marker for a subset of malignancies, but may also be associated with tumor progression in diverse types of human cancers. As the previous study partially demonstrated, it remains to be investigated whether upregulation of podoplanin 
expression might promote the metastatic spread in tumor xenograft model.

While this manuscript was in preparation, the analyses of podoplanin expression by immunohistochemistry using other anti-human podoplanin antibodies was reported $(15,19)$. Interestingly, the commercially available mAb D2-40 used for detecting lymphatic vessels was found to recognize the podoplanin protein. The staining pattern of D2-40 is largely similar to that of 7B10. On the other hand, D2-40 revealed stained bile ducts in the liver, whereas 7B10 did not. Although the reason why our results differ from theirs is not clear, the differences in the sensitivity and specificity of these antibodies may be related to the results. The discrepancy between the antibodies in the immunostaining of cancer cells remains to be investigated. Nevertheless, 7B10 would be also useful for therapeutics because it reacted with native podoplanin protein in flow cytometry.

The research field of lymphangiogenesis is now progressing rapidly. The identification of three lymphatic endothelial markers, LYVE-1 $(28,29)$, podoplanin and prox 1 (30), has recently enabled the precise observation of lymphatic vessels. LYVE-1 was reported to be also expressed by blood vessels in the liver, lung and in some tumors. In contrast, homeobox transcriptional factor, prox 1 was shown to be the master gene for the differentiation of lymphatic endothelial cells. At present, it is the most reliable marker for lymphatics. However, we hardly detect the lymphatic structure by immunostaining for prox 1 , because it is localized at the nucleus of lymphatic endothelial cells. Our results reveal that podoplanin, recognized by $7 \mathrm{~B} 10$, is a specific marker for human lymphatic vessels in both physiological and pathological conditions. Although podoplanin is also expressed by a variety of non-endothelial cells, double stains for podoplanin and CD31 or VEcadherin could be used for the detection of lymphatic vessels. The novel anti-human podoplanin mAb 7B 10 established here, available for immunostaining, would represent a solid contribution for more widespread studies of lymphangiogenesis. In addition, 7B10 can be used for flow cytometry, and thus for isolating several podoplanin positive cells, such as lung type I alveolar cells and mesenchymal cells with unknown functions (unpublished data), as well as lymphatic endothelial cells, raising the possibilities of 7B10 being useful for other research fields.

\section{Acknowledgments}

We thank Drs Naoko Kamo and Kenji Shimizu for helpful discussion. This study was supported by grants from the Ministry of Education, Culture, Sports, Science and Technology, the special Coordination Funds for promoting Science and Technology, the Ichiro Kanehara Foundation, Takeda Science Foundation and the princess Takamatsu Cancer Research Fund.

\section{References}

1. Breiteneder-Geleff S, Soleiman A, Kowalski H, et al: Angiosarcomas express mixed endothelial phenotypes of blood and lymphatic capillaries: podoplanin as a specific marker for lymphatic endothelium. Am J Pathol 154: 385-394, 1999.
2. Saharinen P, Tammela T, Karkkainen MJ, et al: Lymphatic vasculature: development, molecular regulation and role in tumor metastasis and inflammation. Trends Immunol 25: 387-395, 2004.

3. Ramirez MI, Millien G, Hinds A, et al: T1alpha, a lung type I cell differentiation gene, is required for normal lung cell proliferation and alveolus formation at birth. Dev Biol 256: 61-72, 2003

4. Schacht V, Ramirez MI, Hong YK, et al: T1alpha/podoplanin deficiency disrupts normal lymphatic vasculature formation and causes lymphedema. EMBO J 22: 3546-3556, 2003.

5. He Y, Rajantie I, Ilmonen M, et al: Preexisting lymphatic endothelium but not endothelial progenitor cells are essential for tumor lymphangiogenesis and lymphatic metastasis. Cancer Res 64: 3737-3740, 2004.

6. Takahashi M, Yoshimoto T and Kubo H: Molecular mechanisms of lymphangiogenesis. Int J Hematol 80: 29-34, 2004.

7. Shimizu K, Kubo H, Yamaguchi K, et al: Suppression of VEGFR-3 signaling inhibits lymph node metastasis in gastric cancer. Cancer Sci 95: 328-333, 2004.

8. Honn KV, Tang DG and Crissman JD: Platelets and cancer metastasis: a causal relationship? Cancer Metastasis Rev 11: 325-351, 1992.

9. Oleksowicz L and Dutcher JP: Adhesive receptors expressed by tumor cells and platelets: novel targets for therapeutic antimetastatic strategies. Med Oncol 12: 95-102, 1995.

10. Tsuruo T, Yamori T, Naganuma K, et al: Characterization of metastatic clones derived from a metastatic variant of mouse colon adenocarcinoma 26. Cancer Res 43: 5437-5442, 1983.

11. Watanabe M, Okochi E, Sugimoto Y, et al: Identification of a platelet-aggregating factor of murine colon adenocarcinoma 26 : Mr 44,000 membrane protein as determined by monoclonal antibodies. Cancer Res 48: 6411-6416, 1988.

12. Watanabe M, Sugimoto $\mathrm{Y}$ and Tsuruo T: Expression of a $\mathrm{Mr}$ 41,000 glycoprotein associated with thrombin-independent platelet aggregation in high metastatic variants of murine B16 melanoma. Cancer Res 50: 6657-6662, 1990.

13. Sugimoto Y, Watanabe M, Oh-Hara T, et al: Suppression of experimental lung colonization of a metastatic variant of murine colon adenocarcinoma 26 by a monoclonal antibody 8 F11 inhibiting tumor cell-induced platelet aggregation. Cancer Res 51: $921-925,1991$.

14. Toyoshima M, Nakajima M, Yamori T, et al: Purification and characterization of the platelet-aggregating sialoglycoprotein gp44 expressed by highly metastatic variant cells of mouse colon adenocarcinoma 26. Cancer Res 55: 767-773, 1995.

15. Kato Y, Fujita N, Kunita A, et al: Molecular identification of Aggrus/T1alpha as a platelet aggregation-inducing factor expressed in colorectal tumors. J Biol Chem 278: 51599-51605, 2003.

16. Kato Y, Sasagawa I, Kaneko M, et al: Aggrus: a diagnostic marker that distinguishes seminoma from embryonal carcinoma in testicular germ cell tumors. Oncogene 23: 8552-8556, 2004.

17. Ordonez NG: D2-40 and podoplanin are highly specific and sensitive immunohistochemical markers of epithelioid malignant mesothelioma. Hum Pathol 36: 372-380, 2005.

18. Kato Y, Kaneko M, Sata M, et al: Enhanced expression of Aggrus (T1alpha/podoplanin), a platelet-aggregation-inducing factor in lung squamous cell carcinoma. Tumour Biol 26: 195-200, 2005.

19. Schacht V, Dadras SS, Johnson LA, et al: Up-regulation of the lymphatic marker podoplanin, a mucin-type transmembrane glycoprotein, in human squamous cell carcinomas and germ cell tumors. Am J Pathol 166: 913-921, 2005.

20. Kubo H, Fujiwara T, Jussila L, et al: Involvement of vascular endothelial growth factor receptor- 3 in maintenance of integrity of endothelial cell lining during tumor angiogenesis. Blood 96: 546-553, 2000.

21. Kubo H, Cao R, Brakenhielm E, et al: Blockade of vascular endothelial growth factor receptor-3 signaling inhibits fibroblast growth factor-2-induced lymphangiogenesis in mouse cornea. Proc Natl Acad Sci USA 99: 8868-8873, 2002.

22. Mouta Carreira C, Nasser SM, Di Tomaso E, et al: LYVE-1 is not restricted to the lymph vessels: expression in normal liver blood sinusoids and down-regulation in human liver cancer and cirrhosis. Cancer Res 61: 8079-8084, 2001.

23. Morisada T, Oike Y, Yamada Y, et al: Angiopoietin-1 promotes LYVE-1-positive lymphatic vessel formation. Blood 105: 4649-4656, 2005. 
24. Breiteneder-Geleff S, Matsui K, Soleiman A, et al: Podoplanin, novel 43-kd membrane protein of glomerular epithelial cells, is down-regulated in puromycin nephrosis. Am J Pathol 151: 1141-1152, 1997.

25. Nose K, Saito $H$ and Kuroki T: Isolation of a gene sequence induced later by tumor-promoting 12-O-tetradecanoylphorbol13-acetate in mouse osteoblastic cells (MC3T3-E1) and expressed constitutively in ras-transformed cells. Cell Growth Differ 1: 511-518, 1990.

26. Boucherot A, Schreiber R, Pavenstadt H, et al: Cloning and expression of the mouse glomerular podoplanin homologue gp38P. Nephrol Dial Transplant 17: 978-984, 2002.
27. Farr AG, Berry ML, Kim A, et al: Characterization and cloning of a novel glycoprotein expressed by stromal cells in T-dependent areas of peripheral lymphoid tissues. J Exp Med 176: 1477-1482, 1992.

28. Banerji S, Ni J, Wang SX, et al: LYVE-1, a new homologue of the CD44 glycoprotein, is a lymph-specific receptor for hyaluronan. J Cell Biol 144: 789-801, 1999.

29. Prevo R, Banerji S, Ferguson DJ, et al: Mouse LYVE-1 is an endocytic receptor for hyaluronan in lymphatic endothelium. J Biol Chem 276: 19420-19430, 2001.

30. Wigle JT and Oliver G: Prox 1 function is required for the development of the murine lymphatic system. Cell 98: 769-778, 1999. 